\title{
Diagnostic antigens for visceral leishmaniasis: clarification of nomenclatures
}

Tapan Bhattacharyya*, Tegwen Marlais and Michael A. Miles

\begin{abstract}
Background: Stimulated by the increasing recent use of ' $K$ ' or ' $r K$ ' nomenclature for antigens reported for visceral leishmaniasis (VL) diagnostic serology, we wished to give a chronological synopsis of their reporting and the potentially confusing terminology.

Methods: The literature was examined for ' $K$ ' or ' $r K$ ' terminology for VL diagnostic antigens, with emphasis on the original publications in which terms were first used.

Results: A chronological account of the first use of these ' $K$ ' and ' $r K$ ' nomenclatures was compiled. Since the original use of this terminology in 1993 in the name rK39 for a Leishmania antigen fragment, we found nine subsequent instances where ' $\mathrm{K}$ ' or ' $\mathrm{rK}$ ' have been used to maintain consistency with this nomenclature. We also found instances where there were ambiguities regarding reported strain name, origin and GenBank accession numbers.

Conclusions: We have documented here the uses in the literature of the ' $\mathrm{K}$ ' or ' $r \mathrm{~K}$ ' prefix for $\mathrm{VL}$ diagnostic antigen nomenclature. We suggest that, to avoid confusion, the use of such nomenclature for future antigens should either provide the logical derivation of the term or indicate that the designation is entirely empirical.
\end{abstract}

Keywords: Leishmania, Visceral leishmaniasis, Serology, Antigens, Kinesin, Nomenclature, HASPB, Diagnostics, rK39, rK28

\section{Background}

We wish to clarify the potentially confusing ' $\mathrm{K}$ ' or ' $\mathrm{rK}$ ' nomenclature of the antigens used for visceral leishmaniasis (VL) diagnostic serology, by giving a synopsis of their discovery and naming. This has been stimulated by the increasing recent use of this terminology, as listed in Table 1. We therefore focus here on a chronological account of the first use of these nomenclatures rather than an assessment of the use of the antigens in serology or their native function, which are beyond the scope of the current article. By way of introduction it is pertinent to note that Kuhls et al. [1] demonstrated that Leishmania chagasi, the name that had been used for the agent of VL in South America, is synonymous with $L$. infantum deriving from Europe. Thus, the term $L$. infantum (syn. chagasi) will be used where appropriate.

\footnotetext{
* Correspondence: tapan.bhattacharyya@lshtm.ac.uk

Department of Pathogen Molecular Biology, Faculty of Infectious and

Tropical Diseases, London School of Hygiene and Tropical Medicine, London, United Kingdom
}

(c) The Author(s). 2017 Open Access This article is distributed under the terms of the Creative Commons Attribution 4.0 International License (http://creativecommons.org/licenses/by/4.0/), which permits unrestricted use, distribution, and reproduction in any medium, provided you give appropriate credit to the original author(s) and the source, provide a link to the Creative Commons license, and indicate if changes were made. The Creative Commons Public Domain Dedication waiver (http://creativecommons.org/publicdomain/zero/1.0/) applies to the data made available in this article, unless otherwise stated.

\section{Results}

In 1993, in a seminal publication, Burns et al. [2] used a genomic library from a Brazilian strain of $L$. infantum (syn. chagasi) to identify a kinesin-related gene having high specificity and sensitivity in VL serology. A fragment of this gene, encoding a 46 amino acid region followed by $6.5 \times 39$ aa repeats, was expressed as a recombinant protein in E. coli and called rK39 (Fig. 1), where the prefix letter $r$ stands for recombinant.

The following year, a previously identified lipophosphoglycan-associated protein in $L$. donovani entered the literature as KMP-11 (kinetoplastid membrane protein [3]). We have included this antigen in this review because it has been often reported 
Table 1 Chronology of the naming of ' $K$ ' or 'rK' antigens used for serological diagnosis of visceral leishmaniasis

\begin{tabular}{|c|c|c|c|c|c|c|c|}
\hline Year & Reference & Antigen & Species & Parent protein & Reported origin & Strain details & $\begin{array}{l}\text { GenBank accession } \\
\text { number }\end{array}$ \\
\hline 1993 & Burns et al. [2] & rK39 & $\begin{array}{l}\text { L. infantum } \\
\text { (syn. L. chagasi) }\end{array}$ & $\begin{array}{l}\text { LcKin } \\
\text { (kinesin-related) }\end{array}$ & Brazil & $\mathrm{MHOM} / \mathrm{BR} / 82 / \mathrm{BA}-2, \mathrm{C} 1$ & L07879 \\
\hline 1994 & Tolson et al. [3] & KMP-11 & L. donovani & $\begin{array}{l}\text { Kinetoplastid } \\
\text { membrane protein }\end{array}$ & Not given & $\begin{array}{l}\text { LD3, derivative of } \\
\text { 1S2D clone }\end{array}$ & $S 77039^{a}$ \\
\hline 1999 & Alce et al. [4] & $\begin{array}{l}\text { HASPB1/ } \\
\text { HASPB2 }\end{array}$ & L. donovani & HASP & Ethiopia & $\begin{array}{l}\text { MHOM/ET/67/L28 } \\
\text { isolate LV9 }\end{array}$ & $\begin{array}{l}\text { AJ011810/ } \\
\text { AJ011809 }\end{array}$ \\
\hline 1999 & Bhatia et al. [5] & K9/K26 & $\begin{array}{l}\text { L. infantum } \\
\text { (syn. L. chagasi) }\end{array}$ & $\begin{array}{l}\text { Hydrophilic protein } \\
\text { (see text) }\end{array}$ & Brazil & MHOM/BR/74/PP75 & $\begin{array}{l}\text { AF131227/ } \\
\text { AF131228 }\end{array}$ \\
\hline 2006 & Sivakumar et al. [6] & Ld-rKE16 & L. donovani & Kinesin & India & MHOM/IN/98/KE16 & AY615886 \\
\hline 2007 & Gerald et al. [7] & LdK39 & L. donovani & Kinesin LdK39 & Sudan & $\mathrm{MHOM} / \mathrm{SD} / 62 / 1 \mathrm{~S}-\mathrm{CL} 2 \mathrm{D}$ & DQ831678 \\
\hline 2007 & Takagi et al. [9] & rKRP42 & L. donovani & Kinesin & India/Bangladesh & MHOM/IN/80/DD8 & AB256033 \\
\hline 2010 & Pattabhi et al. [8] & rK28 & L. donovani & $\begin{array}{l}\text { HASPB1/LdK39/ } \\
\text { HASPB2 }\end{array}$ & Sudan/Ethiopia & Synthetic gene & HM594686 \\
\hline 2013 & Abass et al. [10] & rKLO8 & L. donovani & Kinesin & Sudan & Lo8 & KC788285 \\
\hline 2015 & Vallur et al. [11] & rK18 & Not given & Not given & Not given & Not given & Not given \\
\hline 2016 & Vallur et al. [12] & rKR95 & L. donovani & $\begin{array}{l}\text { Kinesin-related } \\
\text { protein }\end{array}$ & Bangladesh & Not applicable; see text. & Gl112293604 \\
\hline
\end{tabular}

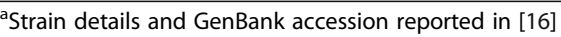

in the literature, but in this case the $\mathrm{K}$ initial was not given to maintain a $\mathrm{K}$ nomenclature.

In 1999, Alce et al. reported the identification and antigenicity of two gene products, named HASPB1 and HASPB2 (for hydrophilic acylated surface protein B), from an Ethiopian strain of $L$. donovani [4]. However, in the same year and the same journal, Bhatia et al. independently reported the characterisation of two hydrophilic antigens, from $L$. infantum (syn. chagasi), which they named K9 and K26 [5]. There are two crucial considerations regarding the description of these latter two antigens: (i) the authors adopted the letter ' $K$ ' prefix in order to maintain consistency with K39; (ii) their report also refers to the identification in GenBank of homologous sequences from $L$. donovani, namely those identified by Alce and colleagues, and thus K9 corresponds to HASPB2, and K26 to HASPB1.

In 2006 and 2007, the first kinesin sequences from South Asian (Indian) and East African (Sudanese) L. donovani $(\mathrm{Ld})$ strains were reported as Ld-rKE16 and LdK39, respectively $[6,7]$. Note that the 'KE' in Ld-rKE16 refers to the given strain name, and not the two-letter abbreviation for Kenya. The first two 39 amino acid repeats of the Sudanese homologue LdK39 were later incorporated into a synthetic gene, where they were flanked by the repeat sequences of HASPB1 and the whole open reading

\section{a}

rK39 from LcKin (L07879)

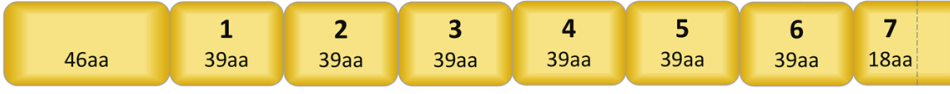

b

rK28 synthetic fusion gene (HM594686)

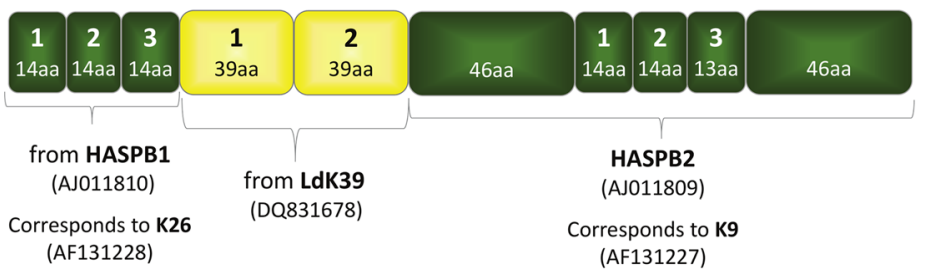

Fig. 1 Schematic representations of VL diagnostic antigens with GenBank accession numbers: a rK39; b synthetic fusion rK28 and its components. Kinesin-derived sequences are depicted in yellow shades, to indicate their different species origin; HASPB sequences depicted in green. Numbering in bold refers to the order of the respective repeat region in the parent protein. Abbreviation: aa, amino acid 
frame of HASPB2, identified by Alce and colleagues (Fig. 1). This new construct was named rK28, further maintaining the letter $\mathrm{K}$ nomenclature of $L$. donovani antigens useful in diagnostic VL serology, and the prefix ' $r$ ' denoting a recombinant protein [8].

The K prefix has also been used for more reported antigens, namely rKRP42 [9], rKLO8 [10], rK18 [11] and rKR95 [12]. rKRP42: derived from strain DD8, which was described by the authors as being from Bangladesh but is listed as World Health Organisation (WHO) reference strain $\mathrm{MHOM} / \mathrm{IN} / 80 / \mathrm{DD} 8$ originating from India $[13,14]$. rKLO8: described by the authors as deriving from Sudanese strain Lo8 (lower case letter o) without full WHO code, but a strain with WHO code MHOM/IN/??/Lo8 [sic], had previously been reported also with a lower case letter o but stating an Indian origin [15]. rK18: no sequences or derivations were given. rKR95: identified from mass spectrometry data of Bangladeshi serum and urine and given an accession number (see Table 1). However, searching this number in GenBank (without the sequence identifier prefix 'GI') retrieved the entry for LdK39, which was submitted by Gerald et al. 2007 and the sequences of which have been used for the kinesin repeats of rK28 $[7,8]$.

\section{Conclusions}

Our intention here is to document the reporting of VL diagnostic antigens using the letter ' $\mathrm{K}$ ' or ' $\mathrm{rK}$ ' prefix nomenclature. We suggest that, to avoid confusion, the use of such nomenclature for future antigens should either provide the logical derivation of the term, for example, indicating the origin, any known function or protein family or that the designation is entirely empirical.

\section{Abbreviations}

HASP: Hydrophilic acylated surface protein; VL: Visceral leishmaniasis; WHO: World Health Organisation

\section{Acknowledgements}

Not applicable.

\section{Funding}

No specific funding was received for this study, however TB has been funded by EC NIDIAG (contract 260260) and TM by the Sir Halley Stewart Trust. The views expressed within this report are those of the authors and not necessarily those of the Trust. TM received a donation towards institutional fees from the John Henry Memorial Fund.

\section{Availability of data and materials}

Genomic data referred to herein is publicly available via Genbank https:// www.ncbi.nlm.nih.gov/genbank/ using the stated accession numbers.

\section{Authors' contributions}

TB initiated the study. TB and TM wrote and MAM edited the manuscript. All authors read and approved the final manuscript.

\section{Competing interests}

The authors declare that they have no competing interests.
Ethics approval and consent to participate

Not applicable.

\section{Publisher's Note}

Springer Nature remains neutral with regard to jurisdictional claims in published maps and institutional affiliations.

Received: 13 December 2016 Accepted: 29 March 2017

Published online: 13 April 2017

\section{References}

1. Kuhls K, Alam MZ, Cupolillo E, Ferreira GE, Mauricio IL, Oddone R, et al. Comparative microsatellite typing of new world Leishmania infantum reveals low heterogeneity among populations and its recent old world origin. PLoS Negl Trop Dis. 2011;5(6):e1155.

2. Burns JM, Shreffler WG, Benson DR, Ghalib HW, Badaro R, Reed SG. Molecular characterization of a kinesin-related antigen of Leishmania chagasi that detects specific antibody in African and American visceral leishmaniasis. Proc Natl Acad Sci USA. 1993;90(2):775-9.

3. Tolson DL, Jardim A, Schnur LF, Stebeck C, Tuckey C, Beecroft RP, et al. The kinetoplastid membrane protein 11 of Leishmania donovani and African trypanosomes is a potent stimulator of T-lymphocyte proliferation. Infect Immun. 1994;62(11):4893-9.

4. Alce TM, Gokool S, McGhie D, Stager S, Smith DF. Expression of hydrophilic surface proteins in infective stages of Leishmania donovani. Mol Biochem Parasitol. 1999:102(1):191-6.

5. Bhatia A, Daifalla NS, Jen S, Badaro R, Reed SG, Skeiky YA. Cloning, characterization and serological evaluation of K9 and K26: two related hydrophilic antigens of Leishmania chagasi. Mol Biochem Parasitol. 1999; 102(2):249-61.

6. Sivakumar R, Sharma P, Chang KP, Singh S. Cloning, expression, and purification of a novel recombinant antigen from Leishmania donovani. Protein Expr Purif. 2006;46(1):156-65.

7. Gerald NJ, Coppens I, Dwyer DM. Molecular dissection and expression of the LdK39 kinesin in the human pathogen, Leishmania donovani. Mol Microbiol. 2007;63(4):962-79.

8. Pattabhi S, Whittle J, Mohamath R, El-Safi S, Moulton GG, Guderian JA, et al. Design, development and evaluation of rK28-based point-of-care tests for improving rapid diagnosis of visceral leishmaniasis. PLoS Negl Trop Dis. 2010;4(9):e822.

9. Takagi H, Islam MZ, Itoh M, Islam AU, Saifuddin Ekram AR, Hussain SM, et al. Short report: production of recombinant kinesin-related protein of Leishmania donovani and its application in the serodiagnosis of visceral leishmaniasis. Am J Trop Med Hyg. 2007;76(5):902-5.

10. Abass E, Bollig N, Reinhard K, Camara B, Mansour D, Visekruna A, et al. rKLO8, a Novel Leishmania donovani-derived recombinant immunodominant protein for sensitive detection of visceral leishmaniasis in Sudan. PLoS Negl Trop Dis. 2013:7(7):e2322.

11. Vallur AC, Hailu A, Mondal D, Reinhart C, Wondimu H, Tutterrow $Y$, et al. Specific antibody responses as indicators of treatment efficacy for visceral leishmaniasis. Eur J Clin Microbiol Infect Dis. 2015;34(4):679-86.

12. Vallur AC, Reinhart $C$, Mohamath $R$, Goto $Y$, Ghosh P, Mondal $D$, et al. Accurate serodetection of asymptomatic Leishmania donovani infection by use of defined antigens. J Clin Microbiol. 2016;54(4):1025-30.

13. World Health Organization. Technical Report Series 949. Control of the leishmaniases, in WHO Technical Report Series. Geneva: World Health Organization; 2010.

14. International Leishmania Network. Strains: Reference and Labelling. http:// leishnet.net/site/. Accessed 6 Oct 2016.

15. Krobitsch S, Brandau S, Hoyer C, Schmetz C, Hubel A, Clos J. Leishmania donovani heat shock protein 100. Characterization and function in amastigote stage differentiation. J Biol Chem. 1998;273(11):6488-94.

16. Jardim A, Hanson S, Ullman B, McCubbin WD, Kay CM, Olafson RW. Cloning and structure-function analysis of the Leishmania donovani kinetoplastid membrane protein-11. Biochem J. 1995;305(1):315-20. 